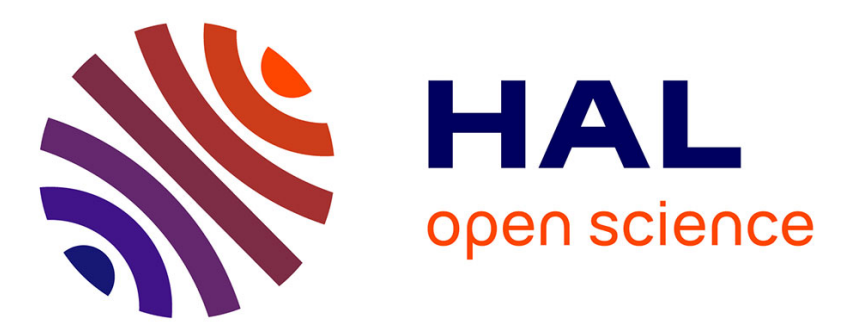

\title{
Vers des compétences externes: l'activisme de la commission européenne en matière d'aviation internationale \\ Cornelia Woll
}

\section{- To cite this version:}

Cornelia Woll. Vers des compétences externes: l'activisme de la commission européenne en matière d'aviation internationale. Politique européenne, 2005, 3 (17), pp.137-158. 10.3917/poeu.017.0137 . hal-01020650

\section{HAL Id: hal-01020650 \\ https: / hal-sciencespo.archives-ouvertes.fr/hal-01020650}

Submitted on 8 Jul 2014

HAL is a multi-disciplinary open access archive for the deposit and dissemination of scientific research documents, whether they are published or not. The documents may come from teaching and research institutions in France or abroad, or from public or private research centers.
L'archive ouverte pluridisciplinaire HAL, est destinée au dépôt et à la diffusion de documents scientifiques de niveau recherche, publiés ou non, émanant des établissements d'enseignement et de recherche français ou étrangers, des laboratoires publics ou privés.

\section{(ㅇ)(1) $\$$}

Distributed under a Creative Commons Attribution - NonCommercial - NoDerivatives| 4.0 


\title{
VERS DES COMPÉTENCES EXTERNES : L'ACTIVISME DE LA COMMISSION EUROPÉENNE EN MATIÈRE D'AVIATION INTERNATIONALE
}

\author{
Cornelia Woll
}

L'Harmattan | Politique européenne

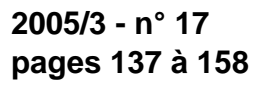

ISSN 1623-6297

Article disponible en ligne à l'adresse:

http://www.cairn.info/revue-politique-europeenne-2005-3-page-137.htm

Pour citer cet article :

Woll Cornelia, « Vers des compétences externes : l'activisme de la commission européenne en matière d'aviation internationale »,

Politique européenne, 2005/3 n 17, p. 137-158.

Distribution électronique Cairn.info pour L'Harmattan.

(c) L'Harmattan. Tous droits réservés pour tous pays.

La reproduction ou représentation de cet article, notamment par photocopie, n'est autorisée que dans les limites des conditions générales d'utilisation du site ou, le cas échéant, des conditions générales de la licence souscrite par votre établissement. Toute autre reproduction ou représentation, en tout ou partie, sous quelque forme et de quelque manière que ce soit, est interdite sauf accord préalable et écrit de l'éditeur, en dehors des cas prévus par la législation en vigueur en France. II est précisé que son stockage dans une base de données est également interdit. 


\section{Cornelia WOLL}

\section{VERS DES COMPETENCES EXTERNES : L'ACTIVISME DE LA COMMISSION EUROPEENNE EN MATIERE D'AVIATION INTERNATIONALE}

Pour analyser le rôle joué par la Commission dans les politiques étrangères européennes, nous montrons comment la Commission peut élargir ses compétences en utilisant ses droits en tant que gardienne des traités. L'étude de cas de la politique européenne en matière d'aviation internationale illustre comment la Commission a pu obtenir un mandat de négociation externe que les Etats membres lui avaient auparavant refusé. Pour cela la Commission s'est appuyée sur des recours juridictionnels et a employé une stratégie cognitive qui inscrit la question de l'aviation civile dans la concurrence avec les Etats-Unis. Par ces deux moyens, la Commission a su changer les préférences des Etats membres et réorienter le point focal des négociations intergouvernementales.

\section{Introduction}

Quel acteur international, l'Union européenne est-elle ? Si l'on veut parler des politiques étrangères européennes ${ }^{1}$ comme étant plus que les actions coordonnées de ses Etats membres, il convient d'analyser le rôle joué par les institutions supranationales². Nous nous proposons d'examiner cette question en étudiant la Commission européenne.

Plusieurs études descriptives ont examiné comment la Commission agit en tant que négociateur externe (Bruter, 1999 ; Nugent, 2001, 297-323 ; Nuttall, 1996). Pourtant, se concentrer uniquement sur son rôle de négociateur externe ne permet pas de

\footnotetext{
${ }^{1}$ Les politiques étrangères européennes couvrent ici toutes les actions politiques de l'Union européenne qui vont au-delà de ses propres frontières. Elles incluent non seulement la Politique européenne de sécurité et de défense, mais aussi les relations économiques et commerciales, l'aide au développement, la coopération régionale, l'élargissement, la promotion des droits de l'homme ou des éléments externes de la politique de l'immigration.

${ }^{2}$ White (2001) effectue la distinction entre les politiques étrangères communautaires (les politiques étrangères de la $\mathrm{CE}$ ), les politiques étrangères de l'Union (les politiques coordonnées des Etats membres) et les politiques étrangères nationales.
}

Politique européenne, $\mathrm{n}^{\circ} 17$, automne 2005, p. 137-158 
comprendre toutes les contributions de la Commission au développement des politiques étrangères. Prétendre que la Commission ne fait que négocier sur la base d'un mandat donné par les Etats membres confirme notamment une vision trop optimiste d'un rapport déléguant-agent (principal-agent) peu problématique : les Etats membres décident de déléguer certaines compétences que la Commission ne fait qu'exécuter sur la scène internationale ${ }^{3}$. En étudiant le cas de l'aviation civile internationale, nous montrerons qu'il faut également examiner comment la Commission peut utiliser son rôle de gardienne des traités pour obtenir des compétences externes que les Etats membres lui avaient auparavant refusées.

En prolongeant l'analyse de l'activisme de la Commission dans l'intégration du marché intérieur de Susanne Schmidt (2000), notre étude de cas montre comment la défense du marché de l'aviation intra-européen a offert à la Commission une marge de manœuvre pour changer la «condition de défaut» du vote des Etats membres en matière internationale. En tant que gardienne des traités, la Commission a su s'appuyer sur la Cour de Justice des Communautés européennes (CJCE) pour mettre en question le cadre existant. Parallèlement à cette stratégie juridique, la Commission a poursuivi une stratégie cognitive et rhétorique pour encadrer la discussion sur l'enjeu de la concurrence américaine. Cette opposition lui a permis de construire une identité européenne en matière d'aviation qui s'est avérée très importante pour la recherche d'un consensus. La Commission n'exécute donc pas seulement la volonté des Etats membres sur la scène internationale, elle promeut aussi indépendamment l'extension de ses propres compétences externes.

L'analyse repose sur 26 entretiens semi-directifs effectués avec des représentants de gouvernements et des compagnies aériennes ainsi que des experts en matière d'aviation aux Etats-Unis et dans l'Union européenne entre septembre 2002 et octobre 20035. Pour clarifier

\footnotetext{
3 Pour un traitement nuancé du conflit principal-agent dans la gouvernance européenne et de la transformation des rapports entre Commission et Etats membre, voir Pollack (2003) et Kassim et Menon (2003).

${ }^{4}$ La condition de défaut est le scénario de base d'un problème public en cas de nondécision. D'habitude, il s'agit du statut quo, mais ce scénario peut également être un changement : par exemple, la libéralisation d'un secteur entamée pour d'autres raisons, qui continuera si une réforme n'est pas votée.

${ }^{5}$ Ces entretiens d'environ une heure et demi incluent l'administration américaine, le Congrès américain, la Commission européenne et sa délégation à Washington, D.C., des représentants des gouvernements allemands, français et britannique, l'American Air Transport Association, l'Airline Pilots Association, l'Association of European
} 
l'argumentation, nous nous interrogeons dans une première partie sur les compétences de la Commission en matière de politiques étrangères. Nous nous tournons ensuite vers l'étude de l'aviation civile internationale. Une présentation du régime international de transport aérien identifie le rôle joué par les Etats dans ce domaine, même après l'intégration du marché intra-européen. Dans la continuité de cette logique traditionnelle, les Etats membres de l'UE ont toujours refusé de céder des compétences de négociations externes à la Commission. Un examen des rapports transatlantiques nous amène ensuite à l'analyse de la stratégie de la Commission pour surmonter l'opposition des Etats membres. En particulier, nous nous intéressons à la boite à outils juridiques et cognitifs de la Commission, pour montrer comment elle a pu s'appuyer sur les arrêts de la Cour de justice européenne et réorienter les enjeux du débat autour de la question transatlantique. La conclusion évalue les généralisations que l'on peut tirer de cette étude de cas.

\section{Le rôle de la Commission dans les politiques étrangères européennes}

La Commission européenne remplit une multitude de fonctions de l'élaboration des politiques à l'administration, la médiation, le suivi et la représentation extérieure (Cini, 1996, 18-33 ; Nugent, 2001, 10$15)$ - ce que Dimitrakopoulos $(2004,1)$ divise en rôles systémiques et sous-systémiques. Les rôles systémiques contribuent au maintien du système de gouvernement européen et couvrent les pouvoirs de la Commission en tant que (a) gardien de la législation européenne et (b) négociateur externe. Des rôles sous-systémiques renvoient à la production politique et incluent l'élaboration des directives ou le suivi de leur mise en œuvre.

Le pouvoir de négociateur externe a suscité beaucoup d'intérêt dans les études de l'UE comme acteur international, d'autant plus que le champ d'intervention de la Commission a augmenté rapidement ces dernières années (Nugent et Saurugger, 2002). Si le Traité de Rome a uniquement donné à la Commission le droit de négocier la politique commerciale commune pour les Etats membres, elle intervient aujourd'hui non seulement sur un échantillon de plus en plus large d'enjeux commerciaux, mais aussi dans des négociations de

Airlines, United Airlines, Delta et American Airlines, Lufthansa, Air France, British Airways et KLM, ainsi que des représentants de l'Organisation Mondiale du Commerce et l'OCDE. 
coopération ou d'association des pays tiers. Elle est engagée dans le processus d'élargissement, gère différentes parties des programmes humanitaires et de développement, ou négocie pour les Etats membres des éléments extérieurs des politiques communautaires, comme la réadmission des immigrés illégaux (MacLeod, Hendry et Hyett, 1996).

Pourtant, un état des lieux de ses compétences externes ne suffit pas pour comprendre le rôle joué par la Commission dans l'évolution des politiques étrangères, puisqu'il ne permet pas de comprendre comment l'administration européenne a obtenu ces compétences. Comment parvient-elle à élargir le champ de ses responsabilités ? La plupart des études sur l'activisme de la Commission pour élargir ses compétences se concentrent sur le marché intérieur (par exemple Schmidt, 1998), mais les dynamiques sont assez comparables en matière de politiques extérieures. Souvent, la Commission publie des commentaires sur des enjeux hors de ses responsabilités, tente d'établir des liens entre ces enjeux et la politique commerciale, qui est de sa compétence, ou prend des actions externes concernant une politique communautaire (Nugent, 2001, 298 ; Nugent et Saurugger, 2002). On pourrait citer les exemples de l'énergie, de l'environnement (Bretherton et Vogler, 2000) ou de la politique de la concurrence (Damro, 2001). Pour accroître ses compétences, la Commission s'appuie notamment sur les décisions de la Cour de justice européenne. Dans ce contexte, le principe de parallélisme a été très important pour les politiques étrangères: en se référant à une compétence interne de la Commission européenne, la Cour de justice européenne justifie la nécessaire mise en conformité de ses ressources externes (MacLeod, Hendry et Hyett, 1996 ; Nugent, 2001, 298).

Afin de penser plus systématiquement ces tentatives d'élargir ses compétences internationales, il est utile de considérer l'analyse de la marge de manœuvre de la Commission vis-à-vis des Etats membres de Susanne Schmidt (2000 ; 2004). Selon Schmidt, la Commission a deux ressources particulières pour peser sur les négociations du Conseil. Premièrement, en tant que gardienne des traités, la Commission est responsable, voire obligée de défendre l'ordre législatif de l'UE. Quand les Etats membres ne sont pas en conformité avec la loi européenne, la Commission peut initier une procédure d'infraction contre eux qui sera suivie par la Cour de justice européenne (article 227). Deuxièmement, la Commission gère le droit de la concurrence de l'UE (titre VI, chapitre 1). Dans cette fonction, elle peut s'opposer à des entreprises ou des régulateurs nationaux qui ne suivent pas les 
principes du droit de la concurrence européenne : elle peut interdire des cartels, empêcher l'abus de position dominante, interdire la discrimination nationale ou des subventions.

En combinaison avec ses droits d'initiative législative, ces capacités permettent à la Commission, selon Schmidt, de jouer deux stratégies distinctes pour peser sur les négociations du Conseil. D’un côté, elle peut tenter de modifier les préférences des Etats membres en poursuivant une stratégie "diviser et conquérir ». D’un autre côté, elle peut changer la condition de défaut contre laquelle les Etats membres évaluent les alternatives politiques en poursuivant une stratégie du moindre mal.

Dans une stratégie consistant à diviser et conquérir, la Commission utilise ses ressources juridiques pour s'adresser directement aux Etats membres, susceptibles de voter contre une proposition de la Commission, pour les obliger à adapter des éléments de leurs situations nationales. Ce mécanisme a été fréquemment utilisé dans le contexte de la libéralisation des secteurs publics. Par des changements à la marge, la Commission modifie les préférences de certains Etats membres en préparation d'une proposition de réforme plus complète du secteur. «Une fois que les Etats membres en question ont dû s'adapter aux demandes de la Commission et ont donc payé le coût d'une réforme domestique, ils sont eux-mêmes intéressés par une réforme plus extensive qui touche l'ensemble de l'UE » (Schmidt, 2000, 47).

Dans une stratégie du moindre mal, la Commission prépare une transformation en proposant une réforme extensive qui incite les Etats membres à développer des propositions alternatives. Pour prendre l'exemple de la libéralisation de l'électricité, la Commission a menacé de mettre en place une libéralisation asymétrique du marché européen en se servant de ses ressources juridiques. La France, qui auparavant préférait maintenir des monopoles nationaux, s'est vue ainsi contrainte de proposer un contre-projet pour éviter cette asymétrie qui était moins extensive que l'idéal de la Commission, mais beaucoup plus libérale que la situation traditionnelle. La Commission a donc défini la condition de défaut contre laquelle les Etats membres ont évalué les alternatives: dès lors, il n'était plus question de préserver l'ancien régime d'électricité, mais d'éviter une libéralisation asymétrique.

Aux stratégies développées par Schmidt s'ajoute une troisième ressource: la Commission est également un forum qui permet d'élaborer un cadre cognitif, voire des référentiels sectoriels 
(Dimitrakopoulos, 2004, 1 ; Muller, 1995 ; 2004). Cette fonction de forum permet à la Commission de se servir de stratégies rhétoriques. En encadrant les enjeux politiques comme les buts européens, la Commission peut tenter de créer de l'unité entre les objectifs des Etats membres. Le penchant de la Commission à rechercher le consensus politique a souvent été discuté (par exemple Cini, 1996, 28-32). Pourtant, parvenir à un consensus est une tâche difficile quand les intérêts des Etats membres divergent. En revanche, quand les intérêts convergent, il convient de parler d'intérêt commun plutôt que de consensus. Dans le contexte des politiques étrangères, l'enjeu est différent. Les Etats membres peuvent avoir des intérêts divergents entre eux dans un domaine politique, mais converger par rapport à un acteur externe. S'intéresser à construction d'un consensus dans les politiques étrangères européennes implique donc de s'interroger pour savoir comment le point focal des négociations entre Etats membres a été déplacé des différences internes vers l'intérêt commun sur la scène internationale ${ }^{6}$. Les stratégies rhétoriques de la Commission fournissent des indications utiles pour une telle analyse.

Surtout dans le contexte européen, la capacité de se comporter en acteur international dépend en grande partie d'une identité collective ou au moins d'une identité thématique (Sedelmeier, 2004 ; Wendt, 1994). Comme le souligne White $(1999,55)$, «la question clef sera de savoir comment la nouvelle Europe peut développer une identité forte ». Il est vrai que les actions politiques qui se référent à une identité collective peuvent le faire de manière instrumentale ou stratégique. Cette référence peut néanmoins encadrer des actions futures puisqu'elles peuvent obliger certains Etats membres à agir dans la continuité de leurs déclarations passées ou parce qu'elles fournissent un point focal pour des négociations à venir (Schimmelfenig, 2001 ; Sedelmeier, 2003). De plus, dans un contexte de politique étrangère, la Commission peut augmenter le soutien pour ses objectifs, si elle peut se servir d'un autre acteur international contre lequel elle arrive à unir les Etats membres.

En résumé, notre attention porte sur les ressources à la disposition de la Commission pour orienter ou exercer des pressions sur les décisions du Conseil en matière de politique étrangère : les recours juridictionnels et les stratégies rhétoriques. L'étude de cas suivante démontre comment l'usage de ces deux éléments a permis à

6 Selon Garrett et Weingast (1993), un enjeu qui permet de structurer des négociations entre des acteurs ayant des intérêts divers peut fournir un "point focal », qui en optique est l'endroit de l'œil où les lumières forment une image. 
la Commission d'obtenir un mandat de négociation dans le domaine de l'aviation internationale en juin 2003, mandat qui lui avait été auparavant refusé.

\section{La logique nationaliste du système bilatéral d'aviation civile}

Afin de comprendre les positions des Etats membres en matière d'aviation civile, il est nécessaire d'examiner l'évolution du système bilatéral qui régit l'aviation internationale. Le régime actuel fut mis en place en 1944 et se fonde sur de multiples accords aériens bilatéraux négociés entre Etats qui constituent aujourd'hui un réseau dense d'arrangements réglementaires. Pour les compagnies aériennes, ce réseau dense est décisif : depuis 1944 plus de 2000 accords de services ont été signés; s'il l'on inclut tous les échanges écrits et les modifications, on arrive même à un chiffre voisin de $10000^{7}$. Les droits de trafic négociés entre les gouvernements nationaux dans ces accords couvrent un grand nombre de détails comme les destinations desservies, les routes et les fréquences des vols, le type d'opération, les capacités de l'avion, les prix et les conditions des billets, la désignation des compagnies et les responsabilités de la propriété et du contrôle des compagnies. Ce dernier élément est un des plus important, puisque la clause relative à la propriété et au contrôle, souvent appelée la clause de nationalité, impose qu'une compagnie soit effectivement entre les mains du pays qui la désigne ${ }^{8}$. Sous le régime bilatéral, aucune compagnie aérienne ne peut prendre des décisions commerciales simples comme l'augmentation de son offre, le choix d'une nouvelle destination, la sollicitation d'investissement étranger important ou la relocalisation de son siège principal.

Auparavant, ce régime bilatéral allait de pair avec un contrôle étatique du régime domestique de transport aérien. La plupart des pays ne possédaient qu'une seule compagnie aérienne internationale, qui était soit subventionnée par l'Etat, soit publique. Pendant les années 1960 et 1970, la rigidité et l'inefficacité économique de ces structures réglementaires ont été mises en question aux Etats-Unis. En 1978, le Airline Deregulation Act prévoyait la fin des activités de l'agence réglementaire, le Civil Aeronautic Board, en 1984. La période de déréglementation domestique qui suivit a profondément transformé l'industrie du transport aérien aux Etats-Unis. Inspiré par cette

\footnotetext{
7 Entretien à Bruxelles, le 26 novembre 2002.

8 La propriété effective est définie aux Etats-Unis comme moins de 25\% de propriété étrangère ; dans les pays de l'UE, elle est à moins de 49\%.
} 
nouvelle solution, le Royaume Uni a déréglementé son secteur de transport aérien de façon similaire sous le gouvernement de Margaret Thatcher en 1979. Comme les Pays Bas, le Royaume Uni a toujours eu une politique aérienne moins restrictive que le reste de l'Europe (Kassim, 1996, 112). Dans la plupart des autres pays européens, par contre, le contrôle étatique des compagnies était fermement établi. A travers l'Europe, les gouvernements étaient l'actionnaire principal majoritaire d'une compagnie nationale, porte drapeau, dite "flag carrier» puisqu'elle portait le drapeau de son pays et le représentait à l'extérieur.

L'expérience américaine de déréglementation n'a pas changé ces traditions. Elle a pourtant inspiré des fonctionnaires de l'UE et de certains pays membres plus libéraux, qui ont envisagé l'idée d'un marché commun du transport aérien. Les deux premiers memoranda de la Commission en matière d'aviation de 1979 et 1984 ont été accueillis très froidement par les gouvernements des pays membres et les compagnies européennes. Toutefois, un accord bilatéral entre le Royaume Uni et les Pays Bas met en place en 1984 un système plus ouvert entre ces deux pays : chaque compagnie des deux partenaires peut dès lors poursuivre ses opérations dans chaque pays sans accord supplémentaire. Avec le Royaume Uni et les Pays Bas en faveur de la libéralisation, la Commission continue donc de faire avancer l'idée d'une approche européenne dans ce que O'Reilly et Stone Sweet (1998) appellent une stratégie « de la carotte et du bâton ».

D'une part, la Commission s'appuie sur la décision « Nouvelles Frontières » de la Cour de justice européenne pour agir contre les prix administrés au niveau national par le biais du droit européen de la concurrence ${ }^{9}$. Sur la base de cette décision la Commission contraint les Etats membres qui suivaient des procédures similaires à abandonner leurs réglementations économiques. Cela parait pratiquement impossible, mais la Commission réussit à faire pression et à soutenir les forces de libéralisation qui montaient en puissance à l'intérieur de pays comme la France ou l'Allemagne. D'autre part, des incitations positives étaient également nécessaires tant l'Italie, la Grèce, le Danemark et l'Espagne restaient fermement opposés aux

\footnotetext{
${ }^{9}$ L'arrêt «Nouvelles Frontières » de 1986 annule une décision de justice française contre plusieurs compagnies aériennes privées et agences de voyages qui vendaient des billets bon marché et non approuvés. La décision de la Cour de justice européenne reconnait ce droit à ces agences et compagnies en soulignant que l'administration des prix du Code français de l'aviation civile avait un effet négatif sur la concurrence à l'intérieur de la CEE.
} 
tentatives de la Commission. Avec l'aide de la Commission, les pays favorables à la libéralisation suggèrent un compromis. Les aéroports régionaux des quatre pays seraient exclus pendant la première phase de la libéralisation; elle devrait ensuite être mise en place au milieu des années 1990. Sur la base de ce compromis, le Conseil se met d'accord sur la libéralisation du transport aérien en Europe en 1987 (pour plus d'informations : Holmes et McGowan, 1997 ; Kassim, 1996 ; O'Reilly et Stone Sweet, 1998 ; Staniland, 2003).

Le paquet de 1987 pose les bases du transfert d'autorité en matière d'aviation vers la Communauté. Deux paquets supplémentaires sont ensuite adoptés par un vote à la majorité qualifiée en juillet 1990 et juillet 1992. Ainsi, le marché unique du transport aérien entre les 17 Etats de la zone économique européenne est institué le 1er avril 1997. Le troisième paquet, de loin le plus important, transforme les compagnies nationales en compagnies aériennes de l'UE titulaires d'une licence, disposant d'un droit d'établissement partout au sein de l'espace européen d'aviation (Mawson, 1997). Il libéralise tous les droits de trafic aux compagnies européennes, y compris le droit de cabotage, c'est-à-dire le droit de transporter des passagers ou du fret entre deux villes d'un pays tiers. Toutes les compagnies dont le capital est propriété des individus d'un des Etats membres ont le droit d'obtenir une licence communautaire pour devenir une compagnie européenne. Au sein de la zone économique européenne, le trafic est dès lors sans restriction et les prix ne sont plus soumis à un contrôle national, même si certaines obligations de service public demeurent en place et peuvent être renforcées. Auparavant marché international, la zone européenne tend à ressembler au marché américain depuis 1997. Pourtant les Etats membre de l'UE ont gardé l'autorité sur les négociations extérieures avec des Etats tiers.

\section{La résistance des Etats membres au transfert de compétences externes}

La situation européenne après 1997 est paradoxale. A l'intérieur de l'UE, les compagnies sont des compagnies communautaires titulaires d'une licence pour opérer dans toute l'Europe. A l'extérieur, par contre, elles sont sujettes à des accords bilatéraux du régime international. Une compagnie détenue par des Britanniques peut donc offrir des vols entre Paris et Nice, mais elle n'a pas le droit de desservir des destinations en dehors de l'UE à partir de la France. Les 
accords de service internationaux sont encore négociés par des délégations des Etats membres et contiennent des clauses relatives à la propriété et au contrôle national. Cette situation contradictoire agace ceux intéressés par l'intégration du marché européen qui se plaignent des limites de la libéralisation interne sans réforme parallèle vers l'extérieur. En revanche, les représentants des Etats membres se satisfont dans l'ensemble de cet arrangement et soulignent à plusieurs reprises qu'il est important de maintenir les droits externes dans la responsabilité des Etats individuels.

En effet, la Commission a tenté d'obtenir un mandat externe en matière d'aviation civile, depuis le début de ses travaux sur le transport aérien, et elle a rencontré beaucoup de résistance de la part des Etats membres. En 1984 déjà, la Commission avait identifié les relations externes comme un aspect majeur de la politique communautaire potentielle en matière d'aviation ${ }^{10}$. Les premières propositions de la Commission pour un mandat externe datent de février 1990 et mars 1992. Elles sont refusées par le Conseil en 1993. En avril 1995, la Commission revient sur le sujet et obtient un mandat très limité pour négocier des accords avec des pays comme le Norvège, la Suède et la Suisse. Une fois le marché intérieur libéralisé, la Commission attire l'attention sur les contraintes imposées par les accords bilatéraux, en argumentant que c'est uniquement au niveau communautaire que ces accords pourront être négociés de manière efficace, en conformité avec la loi européenne. Compte tenu du fait que le Conseil n'est pas disposé à un tel transfert de compétence, la Commission tente de se servir de l'article 133 qui lui accorde la compétence en matière commerciale. Cette possibilité est bloquée lors du Conseil de Nice : le Traité de Nice souligne explicitement que les compétences en commerce de service ne s'appliquent pas aux négociations en transport aérien (article 133 (6)). Cependant, le Conseil finit par donner à la Commission un mandat limité pour les négociations des droits auxiliaires sur la base de l'article $80^{11}$.

Dans les années 1990, il y eut peu de changement dans la position des Etats membres vis-à-vis d'un transfert de compétences pour les négociations des droits de trafic. En soulignant que la Commission manque de personnel et d'expérience, les Etats membres restent

\footnotetext{
10 European Commission (1984), "Progress towards the development of a community air transport policy," COM 8472 final.

11 Les droits auxiliaires (soft rights) couvrent des services auxiliaires aux droits de trafic comme les services aéroportuaires ou le marketing. Les droits de trafic (hard rights) renvoient aux mouvements d'un avion entre deux points géographiques.
} 
fermement opposés à un tel changement ${ }^{12}$. Chaque administration nationale dispose d'unités de négociation bilatérale, avec des négociateurs impliqués de longue date. Des 2054 accords de service enregistrés dans le monde en 2002, presque 1500 étaient négociés par les pays de l'UE (House of Lords, 2003). Maintenir ces accords nécessitait des réunions fréquentes avec les délégations partenaires pour réévaluer les fréquences, les désignations ou d'autres questions. La longue expérience et l'expertise juridique des négociateurs nationaux semblent beaucoup plus importantes que les ambitions intégrationnistes de la Commission. Pour certains observateurs, il s'agit d'une «simple quête d'autorité qu'elle n'est pas capable de remplir ni en terme de contenu, ni en terme de personnel $»^{13}$. Suite aux mandats limités qu'elle a reçus, la Direction générale énergie et transport (DG TREN) de la Commission établit un bureau pour les négociations bilatérales dénommé "accords de service et régulation économique ». En 2000, environ six personnes travaillent dans cette unité $^{14}$. De surcroit, il n'y a que peu d'interaction entre les unités nationales et celles de la Commission européenne ce qui n'améliore pas la confiance des Etats membres.

L'opposition des pays membres au début des années 2000 est donc importante, malgré l'intégration du marché intérieur. Mais la Commission travaille sur plusieurs fronts et elle a encore une carte à jouer : l'opposition aux Etats-Unis. Afin de comprendre comment les Etats-Unis sont devenus le centre du débat européen sur les compétences externes en matière d'aviation civile, il convient d'examiner l'évolution récente du transport aérien transatlantique.

\section{La perspective européenne de la libéralisation internationale initiée par les américains}

Durant les années 1990, l'UE n'est pas la seule à ouvrir son marché régional à l'aviation internationale. Au milieu des années 1990, le gouvernement américain entreprend unilatéralement d'atteindre un certain degré de libéralisation par le biais d'accords bilatéraux moins restrictifs, appelés «de ciel ouvert». Auparavant, les compagnies aériennes en difficulté avaient demandé que les soutiens financiers de

\footnotetext{
12 Entretiens avec des fonctionnaires et des représentants de compagnies aériennes de l'UE le 18 et le 27 novembre et le 2 et le 5 décembre 2002.

13 Entretiens avec des fonctionnaires et des représentants de compagnies aériennes des pays membres de l'UE, le 27 novembre et le 2 décembre 2002.

14 Entretien avec un représentant de la Commission européenne, le 21 octobre 2003.
} 
compagnies étrangères soient autorisés (Tarry, 2000). Néanmoins, les alliances précoces entre compagnies telles que Northwest et KLM Royal Dutch Airlines devaient se voir attribuer l'immunité anti-trust pour être en mesure d'opérer. La tolérance du gouvernement américain pour les alliances transfrontières s'inscrit dans un projet politique plus large. Le gouvernement américain commence à négocier avec certains pays pour libéraliser les accords bilatéraux et octroyer l'immunité anti-trust pour une alliance en contrepartie de l'ouverture du marché du pays d'origine de la compagnie (Garrett et Weingast, 1993; Yergin, Vietor et Evans, 2000). Puisque les alliances précoces sont conclues entre pays qui ne possèdent qu'une seule compagnie aérienne internationale, le calcul est avantageux : ce qui était bon pour KLM est bon pour les Pays-Bas et le gouvernement néerlandais estime que le compromis est équitable. Le premier accord de ciel ouvert est signé entre les Etats-Unis et les Pays-Bas en septembre 1992. Après une série d'accords de ciel ouvert avec de petits pays européens, l'étape suivante consista à conclure un accord de ciel ouvert avec l'Allemagne en 1996, avec une immunité antitrust accordée à l'alliance entre United Airlines et Lufthansa.

A la fin de l'année 2002, 86 accords de ciel ouvert avaient été signés, 59 d'entre eux avec les Etats-Unis ${ }^{15}$. Sous un accord de ciel ouvert, les compagnies aériennes peuvent opérer davantage comme des entreprises classiques sans avoir besoin de négociations gouvernementales si elles désirent modifier leurs fréquences ou leurs capacités. Les seules restrictions qui perdurent concernent (1) le droit d'opérer les vols domestiques dans le pays partenaire et (2) l'actionnariat étranger. Autrement dit, seules les compagnies néerlandaises peuvent opérer sous l'accord de ciel ouvert américanonéerlandais.

Alors que les compagnies aériennes européennes bénéficiaient considérablement de leurs alliances avec leurs homologues américaines et des opportunités commerciales offertes par les nouveaux accords de ciel ouvert, des observateurs européens critiquent le biais qu'ils perçoivent en faveur des Etats-Unis dans ces accords $^{16}$. La fragmentation du marché européen est perçue comme

\footnotetext{
15 La plupart des accords de ciel ouvert concernent deux pays. Il existe pourtant un accord multilatéral entre les Etats-Unis, la Nouvelle Zélande, Singapour, Brunei et le Chili de novembre 2000.

${ }_{16}$ Notamment, des étrangers ne peuvent pas être propriétaires de plus de $25 \%$ d'une compagnie américaine ("propriété et contrôle»), ils ne peuvent pas s'établir à l'intérieur des Etats-Unis (" droit d'établissement»), y proposer des vols domestiques («cabotage »), ou louer un avion avec son équipe à une compagnie américaine ("wet
} 
créant un avantage pour les compagnies américaines. Alors que les compagnies aériennes européennes ne peuvent voler que des EtatsUnis vers leur pays d'origine, leurs consœurs américaines peuvent opérer de n'importe quel pays de ciel ouvert de l'Union européenne vers n'importe quel point des Etats-Unis. Les compagnies américaines ont également obtenu le droit de voler d'un pays de ciel ouvert de l'UE vers un autre, ce qui constitue effectivement une forme de cabotage ${ }^{17}$. Bien plus, les compagnies au sein de l'UE ne peuvent fusionner que si les Etats-Unis ne refusent pas d'octroyer les mêmes droits de trafic à la société nouvellement créée. Au cours des dernières décennies par exemple, British Airways et KLM ont envisagé de fusionner à plusieurs reprises. Puisque British Airways est beaucoup plus importante que KLM, la fusion aurait été principalement britannique. L'accord de ciel ouvert avec les Etats-Unis, cependant, spécifiait que les Pays-Bas ne pouvaient désigner qu'une compagnie à $51 \%$ minimum néerlandaise. La nécessaire renégociation de ces accords signifierait que la fusion ne pourrait se réaliser qu'avec l'accord des Etats-Unis, ce qui implique généralement des concessions supplémentaires ${ }^{18}$.

Cette asymétrie perçue a amené les compagnies européennes à réfléchir à de nouvelles approches de la réglementation internationale (Woll, 2003 ; 2004). Aux yeux des compagnies européennes, les accords de ciel ouvert comportaient un biais important en faveur des américains, qui, selon eux, avaient le pouvoir politique de négocier tout à leur avantage ${ }^{19}$. Afin de faire avancer une réforme au delà de la politique américaine de ciel ouvert, l'Association européenne des compagnies aériennes (AEA) a proposé un projet dit Transatlantic Common Aviation Area (TCAA) ${ }^{20}$. La Commission européenne a tout de suite suivi ce projet avec enthousiasme en en faisant son propre objectif politique. Après des discussions à l'intérieur de l'UE, une

leasing»). De plus, des compagnies étrangères n’ont pas accès aux programmes gouvernementaux ("Fly America»). Pour plus d'information, voir House of Lords (2003).

${ }^{17}$ Ce droit qui est effectivement peu utilisé par des compagnies classiques est très utile aux compagnies américaines de fret qui travaillent en Europe.

18 De ce cas particulier, les Etats-Unis auraient voulu renégocier leur accès à l'aéroport de Heathrow à Londres.

${ }^{19}$ Entretiens avec des représentants de compagnies européennes, le 27 novembre et le 2 décembre 2002.

20 Association of European Airlines (1995), "EU External Aviation Relations," Policy Statement, Brussels: Association of European Airlines; Association of European Airlines (1999), "Towards a Transatlantic Common Aviation Area," Policy Statement, Brussels: Association of European Airlines. 
délégation européenne est allée à Washington D.C. en décembre 1999 pour proposer le projet au gouvernement et aux compagnies américaines. Bien que les américains aient été à l'écoute, ils ne furent pas disposés à négocier un projet si ambitieux avec la Commission qui n'avait pas de compétences formelles en matière de relations externes dans le domaine de l'aviation.

\section{Surmonter l'opposition: la boite à outils juridiques et rhétoriques de la Commission}

A la fin des années 1990, la discussion concernant les droits de négociation externe de la Commission semble loin d'être close. Le marché intra-européen de l'aviation vient d'être intégré avec succès et les Etats membres ne semblent pas particulièrement inquiets de l'intégration incomplète imposée par leurs accords bilatéraux en cours. La libéralisation bilatérale sous initiative américaine crée des asymétries en faveur des Etats-Unis, mais les ciels ouverts présentent également de nombreux avantages. Les Etats membres possèdent un personnel compétent qui travaille sur les accords bilatéraux de transport aérien et estiment qu'accorder des droits de négociation externe à la Commission présente des risques sans apporter de valeur ajoutée $^{21}$. Que se passe-t-il alors jusqu'en 2003 ? Pourquoi les Etats membres acceptent-ils après presque 20 ans d'opposition d'octroyer à la Commission des compétences limitées en matière de transport aérien?

La stratégie de la Commission était double ${ }^{22}$. En premier lieu elle utilise ses obligations de gardienne des traités pour initier des procédures d'infraction contre les accords bilatéraux de plusieurs Etats membres. Puis, sachant que les Etats membres sont opposés à l'octroi d'un mandat externe complet, elle utilise l'opposition aux Etats-Unis pour créer un point focal comme consensus européen.

En tant que défenseur du droit communautaire, la Commission affirme que les anciens accords bilatéraux, et en particulier la clause de

\footnotetext{
${ }^{21}$ Entretiens avec des représentants des Etats membres, le 18 et le 27 novembre 2002, le 20 août et le 5 septembre 2003.

22 Contrairement à d'autres domaines publics, les actions en matière d'aviation internationale ne se caractérisent pas par des divisions au sein de la Commission. Un représentant de la Direction générale de la concurrence affirme que la coopération avec la Direction générale énergie et transport est «très intense » puisque nous "partageons les objectifs de la libéralisation et de la concurrence ». Entretien le 10 septembre 2003. Ajoutons que seulement une douzaine de personnes travaillent dans ce domaine jusqu'en 2002, puisque la Commission n'était pas compétente.
} 
nationalité, sont en conflit avec le concept de compagnie européenne institué lors du troisième paquet de libéralisation voté par le Conseil des Ministres. En décembre 1998, la Commission européenne forme sept recours contre les accords ciel ouvert de l'Autriche, de la Belgique, du Danemark, de la Finlande, de l'Allemagne, du Luxembourg et de la Suède et un huitième contre l'accord bilatéral «Bermude II» entre la Grande Bretagne et les Etats-Unis de décembre 1998. En octobre 1999, les Pays Bas décident de se joindre à la procédure pour soutenir les autres Etats membres. Une seconde série de recours arrive ensuite devant la Cour de justice européenne contre des pays ayant conclu des accords de ciel ouvert avec les Etats Unis après cette date. La Commission avance l'argument que les éléments des accords bilatéraux sont déjà couverts par la législation communautaire. Puisque le cadre normatif qui s'applique à l'aviation a tant évolué, la Commission devait avoir une compétence exclusive en matière d'aviation externe.

Parallèlement à cette stratégie juridique, la Commission concentre ses exigences sur les relations transatlantiques uniquement. Lorsque la Commission demande pour la première fois un mandat pour des négociations externes avec les Etats-Unis «tout le monde y fut particulièrement hostile et assez choqué $»^{23}$. Cette proposition gagne cependant en légitimité à travers la proposition de réponse de l'Association des Compagnies Aériennes Européennes (AEA) qui mène par la suite à la proposition de la TCAA. Cette proposition des compagnies est inspirée par le besoin de consolidation au sein de l'UE. Pour la Commission, les exigences de l'AEA soulignent le caractère incomplet du marché intérieur de l'aviation. Même si la déclaration de l'AEA souligne que l'UE a encore à prouver la « valeur ajoutée » d'une solution communautaire, le projet correspond aux intérêts de la Commission européenne. Il est rapidement adopté en tant qu'objectif de la politique de transport. En promouvant la proposition, la Commission affirme publiquement que la politique américaine de ciels ouverts créé une inégalité entre participants ${ }^{24}$. Seul l'unisson des pays européens pourrait aider à dépasser ce déséquilibre. De plus, l'idée de la TCAA permet à la Commission de souligner le besoin d'une nouvelle solution qui va au-delà d'un accord de ciel

\footnotetext{
${ }^{23}$ Entretien avec un représentant de la Commission européenne, le 21 octobre 2003.

${ }^{24}$ Voir par exemple Karel Van Miert (1995) "EU Competition Policy, the USA and the Air Transport Sector," Discours préparé pour la Sabre World Conference, Dallas,septembre,http://europa.eu.int/comm/competition/speeches/text/sp1995_1 60_en.html.
} 
ouvert multilatéral négocié par les Etats membres. En attendant l'arrêt de la Cour de justice européenne, la Commission continue ainsi de préparer un consensus européen. Elle se rend à Washington D.C. pour rencontrer des interlocuteurs américains afin de discuter la proposition d'une TCAA, même si les Américains se montrent peu impressionnés par le projet européen qui leur semble «surdimensionné »25. Face aux doutes des Etats membres et à la résistance des Américains, la DG TREN commissionne une étude sur les bénéfices d'un accord transatlantique auprès d'un consultant américain, le Brattle Group.

Le 5 novembre 2002, la Cour de justice européenne rend son arrêt dans les affaires que lui avait soumises la Commission à l'encontre du premier groupe des Etats membres. Soutenant l'avis de la Commission, l'arrêt considère que la clause de nationalité et d'autres enjeux couverts dans les accords de ciel ouvert relève de la compétence exclusive de la Commission européenne. En revanche, il confirme que la négociation des droits de trafic avec des pays tiers est l'autorité des Etats membres. Dans un premier temps, les deux côtés se sentent ainsi confortés par le jugement. La Commission réussit toutefois à créer un sentiment d'urgence pour faire avancer la question. Dans une première communication du 19 novembre 2002, elle exige des Etats membres de dénoncer les opérations encadrées par les accords de service en question ${ }^{26}$. La demande est beaucoup trop radicale et coûteuse, mais par cette menace, la Commission veut souligner qu'elle doit être prise en compte dans la recherche d'une nouvelle solution.

La question immédiate qui se pose est de savoir comment les clauses de nationalité peuvent être mises en conformité avec la loi européenne. La Commission déclare qu'elle est prête à jouer son rôle ${ }^{27}$, mais le gouvernement américain ne conçoit pas pourquoi cela devrait être le $\operatorname{cas}^{28}$. A leurs yeux, l'arrêt de la Cour de justice

\footnotetext{
25 Entretiens à Washington, le 2 et le 10 avril 2003. Certaines propositions, comme l'harmonisation des droits de concurrence ou un changement dans le statut de la propriété et du contrôle doivent passer par des législations aux Etats-Unis avec peu de chance de réussite.

${ }^{26}$ European Commission (2002), "Communication from the Commission on the consequences of the Court judgment of 5 November 2002 for European Air Transport Policy," COM 2002649 final.

27 European Commission (2002) "Open sky agreements: Commission welcomes European Court of Justice ruling," Press Release IP/02/1609, Brussels, November 5.

28 Shane, Jeffrey N. (2002) "US Official Comments on EU "Open Skies" Ruling," Discours devant l'American Bar Association, Florida, le 8 novembre.
} 
européenne a justement confirmé que la Commission n'est pas compétente pour la négociation externe. Si la clause de la nationalité doit être modifiée pour inclure la notion d'une compagnie européenne, cela doit se faire logiquement entre les Etats européens et le gouvernement américain. Comme la délégation américaine est très ouverte à une renégociation de la clause de nationalité, elle propose donc une réunion entre ses interlocuteurs traditionnels à Paris en février 2004. Mais la Commission refuse d'être ignorée. Sans invitation, un représentant de la DG TREN se rend à la réunion de Paris pour rappeler aux délégations nationales qu'elles n'ont pas autorité pour modifier les clauses de nationalité selon l'arrêt de la Cour de justice européenne : sous l'article 43 du Traité, la clause relative à la propriété et au contrôle est une compétence communautaire. En effet, l'arrêt de la Cour de justice européenne a créé un vrai paradoxe de compétence pour les négociations bilatérales à venir. Alors que les négociations bilatérales sont entre les mains des Etats membres, certaines clauses des accords à négocier sont de la responsabilité de la Commission. Cette ambiguilté empêche les Etats membres de négocier seuls avec les Etats-Unis, mais ne fournit pas une base légale alternative qui permettra à la Commission d'engager des négociations pour l'ensemble des pays membres.

Dans une deuxième communication du 26 février 2003, la Commission répète le besoin d'un mandat communautaire en tempérant sa position agressive et en proposant de séparer les infractions des accords ciel ouvert et la demande d'un mandat plus extensif ${ }^{29}$. Elle distingue clairement plusieurs demandes: (1) un mandat spécifique pour négocier avec les Etats-Unis, (2) un mandat horizontal pour des négociations dans des domaines qui relèvent de compétences communautaires et (3) une procédure pour la transmission de l'information et la coordination des négociations bilatérales avec des pays tiers entre les Etats membres et la Commission.

Entre temps, le Brattle Group publie son rapport relatif à une «zone d'aviation ouverte» entre les Etats-Unis et l'UE (Moselle, Reitz, Robyn et Horn, 2002) - le nom a été changé pour dissocier le projet de l'ancien TCAA. Le rapport est structuré selon les préoccupations américaines, mais il estime que les retombées économiques bénéficieront surtout au côté européen.

29 European Commission (2003), "Communication from the Commission on relations between the Community and third countries in the field of air transport," COM (2003) 0094 final. 
Encore en novembre 2002, des représentants des Etats membres ont exprimé des doutes concernant l'intérêt d'un transfert de compétence :

«Nous ne sommes pas sûrs d'octroyer un mandat pour les négociations externes à la Commission. Nous en avons beaucoup discuté, mais jusqu’à présent la Commission n'a pas réussi à clarifier la valeur ajoutée d'un tel transfert de compétence. Si elle le fait de manière convaincante, nous allons y réfléchir, mais nous attendons toujours $»^{30}$.

Quelques mois plus tard, cette résistance a disparu. Les gouvernements européens deviennent de plus en plus intéressés par le projet, surtout ceux qui ont des accords de ciel ouvert à ce moment là ${ }^{31}$. Même le Royaume Uni soutient le projet collectif, malgré sa réticence traditionnelle à s'engager dans des négociations de libéralisation avec les Américains puisqu'il ne veut pas perdre son accès privilégié à Heathrow ${ }^{32}$. Ce changement rapide s'explique difficilement par les forces du marché d'aviation internationale ou la menace d'une concurrence américaine, puisque les compagnies américaines souffraient encore des événements du 11 septembre 2001. Comment alors comprendre la réorientation des Etats membres ?

Entre novembre 2002 et printemps 2003, plusieurs changements sont devenus évidents. D’un côté, la stratégie judicaire de la Commission a modifié la condition de défaut des négociations externes. Les Etats membres ne pouvaient ni garder leurs anciens accords, ni négocier des modifications en ce qui concerne les clauses de nationalité. De l'autre côté, la stratégie cognitive centrée sur les Etats-Unis a fini par créer une identité européenne en matière d'aviation civile internationale. Face aux déséquilibres des accords ciel ouvert, les compagnies et gouvernements européens ont souhaité renégocier l'architecture internationale, même si la concurrence américaine n'était pas très forte à ce moment précis. Se concentrer sur

\footnotetext{
30 Entretien avec un responsable de transport aérien d'un Etat membre, le 22 novembre 2002.

31 Seuls le Royaume Uni, l'Irlande, l'Espagne et la Grèce n'ont pas conclu d'accords ciel ouvert avec les Etats-Unis.

32 Un fonctionnaire britannique de l'aviation civile souligne: "nous avons eu tellement de mal à négocier nous-mêmes avec les Américains, l'UE ne peut que faire mieux » (entretien, le 20 mai 2003). Un représentant américain partage ce sentiment, «après toute la frustration de négocier avec les Britanniques, ça ne peut pas être pire avec la Commission » (entretien, le 25 avril 2003).
} 
les relations transatlantiques a ainsi permis d'oublier les différences intra européennes qui ont caractérisé l'aviation civile auparavant.

Le 5 juin 2003, le Conseil des ministres décide enfin de donner un mandat pour les négociations externes des droits de trafic, sur la base de la deuxième communication de la Commission après l'arrêt de la Cour de justice européenne. L'objectif du transfert de compétence est d'établir une coordination des négociations plus claire entre le niveau communautaire et les Etats membres et de faciliter un accord avec les Etats-Unis.

\section{Conclusion}

Nous avons étudié l'aviation civile internationale afin de comprendre en quoi la Commission contribue au développement des politiques étrangères européennes. Au lieu de mettre uniquement en œuvre les compétences que les Etats membres lui ont déléguées, la Commission a également une marge de manœuvre pour élargir son champ de compétences. Conformément à l'analyse du marché intérieur de Schmidt (2000), l'accroissement des compétences externes est le résultat de stratégies juridiques. Cela ne veut pas dire que toutes les stratégies juridiques auront toujours du succès. La tentative de la Commission de s'appuyer sur son mandat en matière commerciale fut sans succès, tout comme ses compétences en matière de concurrence n'ont servi qu'à l'intégration du marché intra-européen. En revanche, son droit de lancer une procédure d'infraction en combinaison avec le recentrage de l'enjeu sur les Etats-Unis a fini par amener le Conseil à lui transférer un mandat de négociation internationale le 5 juin 2003.

En tant que gardienne des traités, la Commission a su créer de l'incertitude légale concernant la procédure à suivre. De surcroît, son statut de forum lui a permis d'alimenter un consensus autour d'une proposition concrète qui se concentrait sur les relations transatlantiques. Le premier de ces deux éléments a eu un effet sur la condition de défaut contre laquelle les Etats membres ont évalué les alternatives, puisqu'ils n'étaient plus en mesure de poursuivre les procédures traditionnelles, sous peine d'être poursuivis devant la Cour de justice européenne. Le deuxième a apporté un point focal pour réorganiser les préférences des Etats membres autour d'un nouvel enjeu. Même s'ils étaient fermement opposés à un transfert de compétences externes à la Commission, les Etats membres ont pu se mettre d'accord sur le besoin de se réunir pour négocier avec les 
Etats-Unis. Le projet d'une zone d'aviation ouverte était une solution adaptée à ces deux réflexions : il fournissait une réponse à l'incertitude légale toute en créant un consensus européen, voire une identité thématique. Les ressources que la Commission peut saisir dans son interaction avec le Conseil sont donc similaires en matière internationale et en matière d'intégration du marché européen, même si la rhétorique et l'enjeu de l'identité jouent un rôle plus important quand l'UE doit agir vers l'extérieur.

Se basant sur étude monographique, l'application de nos conclusions à d'autres domaines de politiques étrangères doit être avancée avec prudence. Nous ne suggérons pas de façon mécanique que la Commission se servira tonjours des stratégies juridiques ou rhétoriques pour augmenter ses compétences externes. Notre intention est plutôt de mettre en question l'hypothèse selon laquelle cela ne serait jamais le cas et qui justifie des analyses purement institutionnelles des politiques étrangères européennes. L'étude de l'activisme de la Commission permet de montrer les dangers de la délégation et d'apprécier les «expressions variées d'autonomie supranationale» (Pollack, 1997, 101). La Commission agit non seulement suite aux demandes des Etats-membres en matière internationale, elle tente aussi à élargir ses compétences, parfois contre la volonté d'un ou plusieurs Etats-membres. Même si la combinaison particulière des outils employés par la Commission peut varier selon les domaines, une interrogation sur ses capacités potentielles à élargir son champ d'action doit faire partie des analyses institutionnelles du développement des politiques étrangères européennes.

\section{BIBLIOGRAPHIE}

Bretherton, C. et Vogler, J. (2000), "The European Union as Trade Actor and Environmental Activist: Contradictory Roles?" Journal of Economic Integration, 15 (2), p. 163-194.

Bruter, M. (1999), "Diplomacy without a State: the External Delegations of the European Commission", Journal of European Public Policy, 6 (2), p. 183-205.

Cini, M. (1996), The European Commission: Leadership, Organisation and Culture in the EU Administration, Manchester, Manchester University Press.

Damro, C. (2001), "Building an International Identity: the EU and Extraterritorial Competition Policy", Journal of European Public Policy, 8 (2), p. 208-226. 
Dimitrakopoulos, D. (dir.) (2004), The Changing European Commission, Manchester: Manchester University Press.

Garrett, G. et Weingast, B. (1993), "Ideas, Interests and Institutions: Constructing the European Communities Internal Market", dans Ideas and Foreign Policy: Beliefs, Institutions and Political Change, dirigé par J. Goldstein et R. Keohane, Ithaca, N.Y., Cornell University Press.

Holmes, P. et McGowan, F. (1997), "The Changing Dynamic of EU-Industry Relations: Lessons from the Liberalization of European Car and Airline Markets", dans Participation and Policy-Making in the European Union, dirigé par H. Wallace et A. Young, Oxford, Claredon Press.

House of Lords (2003), "'Open Skies' or Open Markets? The Effect of the ECJ Judgements on Aviation Relations between the EU and the USA", London, House of Lords.

Kassim, H. (1996), "Air Transport", dans The European Union and National Industrial Policy, dirigé par H. Kassim et A. Menon, London, Routledge.

Kassim, H. et Menon, A. (2003), "The Principal-Agent Approach and the Study of the EU: Promise Unfulfilled?" Journal of European Public Policy, 10 (1), p. 121-139.

MacLeod, I., Hendry, I. D. et Hyett, S. (1996), The External Relations of the European Communities: a Manual of Law and Practice, Oxford, Clarendon Press.

Mawson, J. (1997), "Air Transport Liberalization in the European Union: An Assessment", Regional Studies, 31 (8), p. 807-822.

Moselle, B. et al. (2002), "The Economic Impact of an EU-US Open Aviation Area", Washington D.C., The Brattle Group.

Muller, P. (1995), "Un espace européen de politiques publiques", dans Politiques publiques en Europe, dirigé par Y. Mény, P. Muller et J.-L. Quermonne, Paris, L'Harmattan.

Muller, P. (2004), "Référentiel", dans Dictionnaire des politiques publiques, dirigé par L. Boussaguet, S. Jacquot et P. Ravinet, Paris, Presses de Science Po.

Nugent, N. (2001), The European Commission, Basingstoke, Palgrave.

Nugent, N. et Saurugger, S. (2002), "Organizational Structuring: the Case of the European Commission and its External Policy Responsibilities", Journal of European Public Policy, 9 (3), p. 345-364.

Nuttall, S. (1996), "The Commission: The Struggle for Legitimacy", dans The Actors in Europe's Foreign Policy, dirigé par C. Hill, London, Routledge.

O'Reilly, D. et Stone Sweet, A. (1998), "The Liberalization and Reregulation of Air Transport", Journal of European Public Policy, 5, (3), p. 441-466.

Pollack, M. A. (1997), "Delegation, Agency and Agenda Setting in the European Community", International Organization, 51, (1), p. 99-134.

Pollack, M. A. (2003), The engines of European integration: delegation, agency, and agenda setting in the EU, Oxford, Oxford University Press. 
Schimmelfenig, F. (2001), "The Community Trap: Liberal Norms, Rhetorical Action, and the Eastern Enlargement of the European Union", International Organization, 55 (1), p. 47-80.

Schmidt, S. K. (1998), "Commission Activism: Subsuming Telecommunication and Electricity under European Competition Law", Journal of European Public Policy, 5 (1), p. 169-184.

Schmidt, S. K. (2000), "Only an Agenda Setter? The European Commission's Power over the Council of Ministers", European Union Politics, 1 (1), p. 37-61.

Schmidt, S. K. (2004), "The European Commission's Powers in Shaping European Policies", dans The Changing European Commission, dirigé par D. Dimitrakopoulos, Manchester, Manchester University Press.

Sedelmeier, U. (2003), "EU Enlargement, Identity and the Analysis of European Foreign Policy: Identity Formation through Policy Practice", EUI Working Papers, 2003, (13).

Sedelmeier, U. (2004), "Collective Identity", dans Contemporary European Foreign Policy, dirigé par W. Carlsnaes, H. Sjursen et B. White, Thousand Oaks, C.A., Sage, p. 123-140.

Staniland, M. (2003), Government Birds: The State and Air Transport in Western Europe, Lanham, M.D., Rowman and Littlefield.

Tarry, S. E. (2000), "Globalization and the Prospect of Policy Convergence in Air Transport", Global Society, 14 (2), p. 279-296.

Wendt, A. (1994), "Collective Identity Formation and the International State", American Political Science Review, 88 (2), p. 384-396.

White, B. (1999), "The European Challenge to Foreign Policy Analysis", European Journal of International Relations, 5 (1), p. 37-66.

White, B. (2001), Understanding European Foreign Policy, London, Palgrave.

Woll, C. (2003), "Transatlantic relations as a catalyst to European integration: the activism of the European Commission in the case of international aviation", AICGS/DAAD Policy Paper Series, Washington, D.C.

Woll, C. (2004), "The Politics of Trade Preferences: Business Lobbying on Service Trade in the United States and the European Union", Thèse soutenue à l'Institut d'Etudes Politiques de Paris et Universität zu Köln.

Yergin, D., Vietor, R. H. K. et Evans, P. C. (2000), "Fettered Flight: Globalization and the Airline Industry", Cambridge M.A., Cambridge Energy Research Associates. 